\title{
Neurofilament Light Chain as a Biomarker of Hereditary Transthyretin-Mediated Amyloidosis
}

Simina Ticau, PhD,* Gautham V. Sridharan, PhD,* Shira Tsour, MS, William L. Cantley, PhD, Amy Chan, PhD, Jason A. Gilbert, MS, David Erbe, PhD, Emre Aldinc, MD, Mary M. Reilly, MD, David Adams, MD, Michael Polydefkis, MD, Kevin Fitzgerald, PhD, Akshay Vaishnaw, MD, and Paul Nioi, PhD

Neurology ${ }^{\circledR}$ 2021;96:e412-e422. doi:10.1212/WNL.0000000000011090

\section{Abstract}

\section{Objective}

To identify changes in the proteome associated with onset and progression of hereditary transthyretin-mediated (hATTR) amyloidosis, also known as ATTRv amyloidosis, we performed an observational, case-controlled study that compared proteomes of patients with ATTRv amyloidosis and healthy controls.

\section{Methods}

Plasma levels of $>1,000$ proteins were measured in patients with ATTRv amyloidosis with polyneuropathy who received either placebo or patisiran in a Phase 3 study of patisiran (APOLLO), and in healthy controls. The effect of patisiran on the time profile of each protein was determined by linear mixed model at 0,9 , and 18 months. Neurofilament light chain (NfL) was further assessed with an orthogonal quantitative approach.

\section{Results}

Levels of 66 proteins were significantly changed with patisiran vs placebo, with NfL change most significant $\left(p<10^{-20}\right)$. Analysis of changes in protein levels demonstrated that the proteome of patients treated with patisiran trended toward that of healthy controls at 18 months. Healthy controls' NfL levels were 4-fold lower than in patients with ATTRv amyloidosis with polyneuropathy $(16.3 \mathrm{pg} / \mathrm{mL}$ vs $69.4 \mathrm{pg} / \mathrm{mL}$, effect $-53.1 \mathrm{pg} / \mathrm{mL}$ [ $95 \%$ confidence interval -60.5 to -45.9]). NfL levels at 18 months increased with placebo $(99.5 \mathrm{pg} / \mathrm{mL}$ vs $63.2 \mathrm{pg} / \mathrm{mL}$, effect 36.3 $\mathrm{pg} / \mathrm{mL}[16.5-56.1])$ and decreased with patisiran treatment $(48.8 \mathrm{pg} / \mathrm{mL}$ vs $72.1 \mathrm{pg} / \mathrm{mL}$, effect $-23.3 \mathrm{pg} / \mathrm{mL}[-33.4$ to -13.1$]$ ) from baseline. At 18 months, improvement in modified Neuropathy Impairment Score +7 score after patisiran treatment significantly correlated with reduced $\mathrm{NfL}(R=0.43[0.29-0.55])$.

\section{Conclusions}

Findings suggest that NfL may serve as a biomarker of nerve damage and polyneuropathy in ATTRv amyloidosis, enable earlier diagnosis of patients with ATTRv amyloidosis, and facilitate monitoring of disease progression.

\section{Classification of Evidence}

This study provides Class III evidence that NfL levels may enable earlier diagnosis of polyneuropathy in patients with ATTRv amyloidosis and facilitate monitoring of disease progression.

\author{
Correspondence \\ Dr. Nioi \\ pnioi@alnylam.com
}

\section{MORE ONLINE}

$\rightarrow$ Class of Evidence

Criteria for rating

therapeutic and diagnostic studies

NPub.org/coe

\footnotetext{
*These authors contributed equally to this work.

From Alnylam Pharmaceuticals (S.T., G.V.S., S.T., W.L.C., A.C., J.A.G., D.E., E.A., K.F., A.V., P.N.) , Cambridge, MA; MRC Centre for Neuromuscular Diseases (M.M.R.), UCL Queen Square Institute of Neurology, London, UK; AP-HP (D.A.), Université Paris-Saclay, CHU Bicêtre, INSERM U1195, Le Kremlin Bicêtre, France; and Department of Neurology (M.P.), Johns Hopkins University School of Medicine, Baltimore, MD.

Go to Neurology.org/N for full disclosures. Funding information and disclosures deemed relevant by the authors, if any, are provided at the end of the article. 


\section{Glossary}

APOLLO = Phase 3 Study of Patisiran for the Treatment of Hereditary Transthyretin-Mediated Amyloidosis With Polyneuropathy; $\mathbf{A T T R v}=$ hereditary transthyretin; CI = confidence interval; CMT = Charcot-Marie-Tooth; hATTR = hereditary transthyretin-mediated; mNIS+7 = modified Neuropathy Impairment Score +7; NfL = neurofilament light chain; NPX $=$ normalized protein expression; NT-proBNP $=$ N-terminal prohormone of brain-type natriuretic peptide; PCA = principal component analysis; PC1 = first principal component; $\mathbf{P N D}=$ polyneuropathy disability; $\mathbf{R N A i}=\mathrm{RNA}$ interference; TTR $=$ transthyretin

Hereditary transthyretin-mediated (hATTR) amyloidosis, also known as ATTRv ( $\mathrm{v}$ for variant) amyloidosis, is a rare, progressively debilitating, and fatal disease caused by pathogenic mutations in the transthyretin (TTR) gene that result in accumulation of amyloid fibrils throughout the body. ${ }^{1,2}$ This accumulation leads to damage of multiple organs and tissues, ${ }^{3}$ and the majority of patients with ATTRv amyloidosis develop a mixed phenotype of both polyneuropathy and cardiomyopathy. ${ }^{4-6}$ Due to variability of the initially affected tissues, age at onset, and penetrance of ATTRv amyloidosis, it is difficult to predict disease onset and progression in individual patients.

Diagnosis of ATTRv amyloidosis remains challenging, ${ }^{7,8}$ and penetrance of the disease in carriers varies widely by region ${ }^{9}$ and mutation. ${ }^{10}$ Modified Neuropathy Impairment Score +7 $(\mathrm{mNIS}+7)$ can be used to assess severity and progression of polyneuropathy in patients with ATTRv amyloidosis, ${ }^{11}$ but while a helpful tool, it can be burdensome to administer.

Due to the low prevalence of ATTRv amyloidosis, a limited number of clinical proteomic studies have been conducted. ${ }^{12,13}$ The pivotal Phase 3 APOLLO study in patients with ATTRv amyloidosis with polyneuropathy (NCT01960348) was a randomized, double-blind, placebo-controlled trial of patisiran, an RNA interference (RNAi) therapeutic. ${ }^{6,14}$ The objective of this study was to use plasma samples collected during APOLLO to evaluate the change in circulating proteins in response to patisiran treatment in patients with ATTRv amyloidosis with polyneuropathy. The clinical utility of biomarkers could include monitoring of disease progression and treatment effect; biomarker development could lead to an early diagnostic predictor of when disease becomes manifest in asymptomatic carriers of pathogenic TTR mutations.

\section{Methods}

The primary research purpose was to evaluate biomarkers that may enable earlier diagnosis of polyneuropathy in patients with ATTRv amyloidosis and facilitate monitoring of disease progression (Class III evidence).

\section{Study Population}

The present case-controlled study includes analysis of plasma samples in a subset of patients enrolled in the APOLLO study who completed the study and consented to a biomarker discovery study. APOLLO eligibility criteria included
Neuropathy Impairment Score of 5 to 130 (range 0-244, with higher scores indicating more impairment) and a polyneuropathy disability (PND) score of IIIb or lower. ${ }^{6}$ During the APOLLO study, patients received either patisiran $0.3 \mathrm{mg} /$ $\mathrm{kg}$ or placebo intravenously every 3 weeks for 18 months. ${ }^{6}$ Further details of the APOLLO methodology have previously been described. ${ }^{6}$ Healthy control samples $(n=57)$ were collected separately and were age, sex, and race matched to the baseline demographics of the APOLLO patients included in this analysis (Dx Biosamples, LLC, San Diego, CA).

\section{Standard Protocol Approvals, Registrations, and Patient Consents}

Plasma samples were taken from patients enrolled in the phase 3 APOLLO study (NCT01960348; ClinicalTrials.gov). ${ }^{6}$ APOLLO was conducted according to the guidelines of the International Conference on Harmonization, the World Health Organization Declaration of Helsinki, and the Health Insurance Portability and Accountability Act of 1996. Written informed consent was obtained from all patients. The APOLLO study protocol and all amendments were approved by the local institutional review boards and ethics committees. Healthy control samples were collected under institutional review board-approved protocols.

\section{Measures}

mNIS+7 is a composite measure of neuropathy that assesses motor, sensory, and autonomic neuropathy (range 0-304, with higher scores indicating more impairment) using a number of standardized assessments to quantify muscle weakness, muscle stretch reflexes, sensory loss, nerve conduction, and autonomic impairment. ${ }^{15}$ It represents the first Neuropathy Impairment Score specifically designed to assess patients with ATTRv amyloidosis in clinical trials. PND score is also used to assess severity of neuropathy in patients with ATTRv amyloidosis, with higher scores indicating greater impairments to ambulation. In the APOLLO study, all patients were assessed for $\mathrm{mNIS}+7$ and PND scores at baseline and 9 and 18 months. ${ }^{6}$

Plasma samples of patients enrolled in APOLLO were collected according to the study protocol before dosing $(n=136$ patisiran-treated, $n=53$ placebo-treated patients). ${ }^{6}$ Levels of 1,161 unique proteins (1,196 total proteins) were measured with 13 human panels containing 92-plex immunoassays (Olink Proteomics, Watertown, MA) with intra-assay coefficients of variation ranging from $2 \%$ to $14 \%$ and interassay 
coefficients of variation ranging from $5 \%$ to $26 \%$. Approximately $10 \%$ of the data points fell below the limit of detection of the assays with $<1 \%$ of proteins not being detected in $>50 \%$ of the 624 samples. Olink uses a proprietary proximity extension assay technology to combine a detection step involving oligonucleotide-labeled antibodies with a proximitydependent DNA polymerization step and a real-time quantitative PCR amplification step to measure relative levels of multiple biomarkers simultaneously. Relative protein levels are reported as normalized protein expression (NPX) values, which are on a $\log _{2}$ scale.

Subsequent quantitative measurements of neurofilament light chain (NfL) were made using an ultrasensitive single molecule array method with intra-assay coefficients of variation $<12 \%$ and interassay coefficients of variation $<10 \%$, in duplicate, in a subset of patients from whom sufficient volume of plasma was available ( $n=112$ patisiran treated, $n=47$ placebo treated) at baseline and 18 months. All NfL values were within the linear ranges of the assay except for 2 measurements, which were excluded.

\section{Statistical Analysis}

The overall study size was determined by the number of patients who completed the APOLLO study and had plasma samples available at the 3 assessment time points. The number of healthy controls was more than double what was needed to detect a $20 \%$ difference in protein levels between healthy controls and baseline APOLLO participants, with $80 \%$ power and $5 \%$ significance to account for variable potential variance of proteins measured and to closely match the patient demographics.

To determine which protein levels changed the most over time as a result of placebo vs patisiran treatment, a linear mixed model regression analysis was performed for each protein to determine whether there was a significant differential time profile based on treatment. The Wilkinson notation representation of the model is given by the following: protein NPX $\sim$ treatment + time + treatment:time + age + sex $+(1 \mid$ patient $)$, in which the response protein NPX represents protein levels, treatment denotes whether the patient was administered placebo or patisiran, time is a numeric quantity representing 0,9 , or 18 months, treatment:time is the interaction term, age and sex are additional covariates, and individual patients (patient) are assigned random intercepts. The model was regressed for each protein using the fit linear mixed-effects model (fitlme) function (MATLAB 9.3; MathWorks, Natick, MA), and the coefficients and $p$ values associated with the treatment:time interaction coefficient were stored and plotted.

Principal component analysis (PCA) is a statistical procedure used to convert a set of observations that may be correlated into a set of linearly uncorrelated values called principal components. This transformation results in the first principal component (PC1) accounting for the largest possible variance in the data, with each subsequent principal component accounting for less variance. Here, the analysis is used to display the levels of 66 proteins in various cohorts of individuals (healthy control, baseline APOLLO, APOLLO/placebo, and APOLLO/ patisiran) on a simple 2-dimensional plot by plotting the 2 principal components that explain most of the variability in the data. Missing measurements were replaced with the limit of detection for PCA analysis.

All boxplots are based on standard metrics, namely the minimum value, maximum value, lower and upper quartiles, and median value. Outliers are denoted as those values that are $>1.5$ times the interquartile range above the third quartile or below the first quartile. Plots were made with the Python Matplotlib graphics environment.

Because NfL has been shown to vary with age, we also report adjustment for this covariate when applicable. All correlation analysis was performed with Pearson correlation and partial correlations for covariate adjustment. All comparisons of means between groups were performed with an unpaired 2-sample $t$ test in R ( R Foundation for Statistical Computing, Vienna, Austria). To adjust for age, intergroup comparisons were tested using linear regressions with age as a covariate. Comparisons between the same individuals over time were not age adjusted because the longest time frame looked at was 18 months.

PCA analysis was performed with the Python sklearn package, and the $99.9 \%$ confidence intervals (CIs) were drawn using the variance-covariance matrix of each treatment group's multivariate distribution using the Ellipse patch as part of Matplotlib. Receiver operating characteristic curves were drawn by plotting the true-positive rate against the falsepositive rate for varying cutoff values. The area under the curve was calculated. The Youden index was used to calculate the NfL level that best discriminates the healthy control cohort from APOLLO patients at baseline.

\section{Data Availability}

The datasets generated and analyzed during the current study are not publicly available.

\section{Results}

Proteomes were compared in 136 patients treated with patisiran and 53 patients treated with placebo with ATTRv amyloidosis with polyneuropathy from the APOLLO study and 57 healthy controls who were age, sex, and race matched to the APOLLO patients (figure 1 and table 1). The APOLLO patient biomarker cohort was demographically representative of the patients enrolled in APOLLO, ${ }^{6}$ and at baseline, patients in the patisiran and placebo groups had similar levels of polyneuropathy severity as measured by mean mNIS +7 score (79.0 [range 8-165] and 74.1 [range 11-151], respectively) and PND score (PND I: $26 \%$ vs $25 \%$, PND II: $32 \%$ vs $28 \%$, PND IIIa: $29 \%$ vs $28 \%$, PND IIIb: $18 \%$ vs $13 \%$ ). 


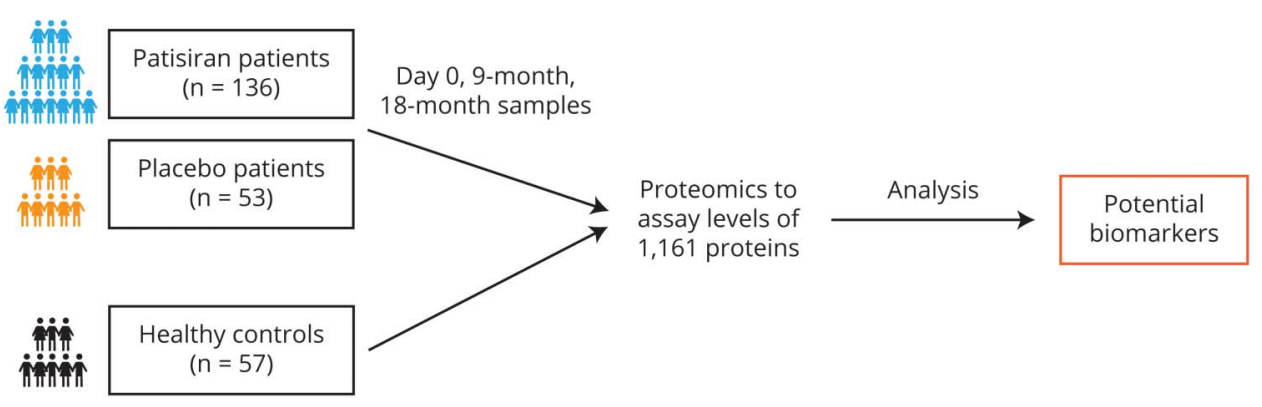

\section{Multiple Plasma Proteins Differ Significantly Between Patients Treated with Placebo and Patisiran}

Plasma levels of 1,161 unique proteins were analyzed with a proximity extension assay. A linear mixed model was used to determine the impact of patisiran treatment on the time profile of the plasma level of each protein by analyzing levels at baseline and 9 and 18 months. A total of 66 proteins were found to show a significant change in levels in patients treated with placebo vs patisiran over time $(p<$ $4.18 \times 10^{-5}$, with Bonferroni correction), of which NfL was the most significant $\left(p=3.95 \times 10^{-21}\right.$, figure 2$)$. This analysis revealed that on treatment with patisiran, levels of some proteins increased (e.g., neutral ceramidase), whereas levels of other proteins decreased (e.g., NfL, R-spondin 3, coiled-coil domain containing 80). Many of the proteins affected by patisiran treatment have not been described as biomarkers (table e-1, doi.org/10.5061/dryad. 1vhhmgqqk); however, 1 previously described biomarker of cardiac health, N-terminal prohormone of brain-type natriuretic peptide (NT-proBNP), showed a significant decrease $\left(p=7.02 \times 10^{-14}\right)$ on patisiran treatment. The NT-proBNP levels predicted by the proximity extension assay data and linear mixed model were consistent with those measured with an orthogonal method in the APOLLO study. ${ }^{6}$

\section{Effect of Disease Progression and Treatment on Overall Plasma Proteome Signature}

PCA was used to elucidate whether there was a systemic plasma proteomic shift in patients with ATTRv amyloidosis with polyneuropathy and whether there was a subsequent reversal after treatment with patisiran. The 66 most significant proteins found in the mixed model analysis (table e-1, doi.org/10.5061/dryad.1vhhmgqqk) were compared in samples from healthy controls and baseline samples from patients with ATTRv amyloidosis with polyneuropathy. Projecting each individual's data onto the first 2 principal components that account for the most variance revealed a distinct separation between healthy controls and patients with ATTRv amyloidosis with polyneuropathy at the APOLLO baseline (figure 3A), with most of the separation being driven by the PC1. The proteomes of patients treated with placebo at 18 months were projected onto the same principal components resulting in a leftward movement along PC1 of the $99.9 \%$ confidence ellipse, suggesting that the proteome of patients treated with placebo was further separating from healthy

Table 1 Baseline Demographics of APOLLO Study Patients Participating in the Biomarker Study and the Age/Sex/Race Matched Healthy Controls

\begin{tabular}{|c|c|c|c|c|c|}
\hline & \multicolumn{3}{|l|}{ APOLLO } & \multicolumn{2}{|c|}{ Healthy controls } \\
\hline & $\begin{array}{l}\text { Placebo (baseline) } \\
(n=53)\end{array}$ & $\begin{array}{l}\text { Patisiran (baseline) } \\
(\mathrm{n}=136)\end{array}$ & $\begin{array}{l}\text { Total } \\
(n=189)\end{array}$ & $n=57$ & $p$ Value \\
\hline Mean age (SD), y & $62.4(11.0)$ & $59.7(12.1)$ & $60.5(11.8)$ & $58.6(12.1)$ & 0.32 \\
\hline Male, n (\%) & $41(77.4)$ & $101(74.3)$ & $142(75.1)$ & $42(73.7)$ & 0.93 \\
\hline \multicolumn{6}{|l|}{ Race, n (\%) } \\
\hline Asian & $15(28.3)$ & $23(16.9)$ & $38(20.1)$ & $12(21.1)$ & 0.90 \\
\hline Black or African American & $0(0)$ & $4(2.9)$ & $4(2.1)$ & $2(3.5)$ & 0.56 \\
\hline White & $37(69.8)$ & 105 (77.2) & $142(75.1)$ & $43(75.4)$ & 0.99 \\
\hline Multiple/other/unknown & $1(1.9)$ & $4(2.9)$ & $5(2.6)$ & $0(0.0)$ & 0.22 \\
\hline
\end{tabular}

Abbreviation: APOLLO = Phase 3 Study of Patisiran for the Treatment of Hereditary Transthyretin-Mediated Amyloidosis with Polyneuropathy. 


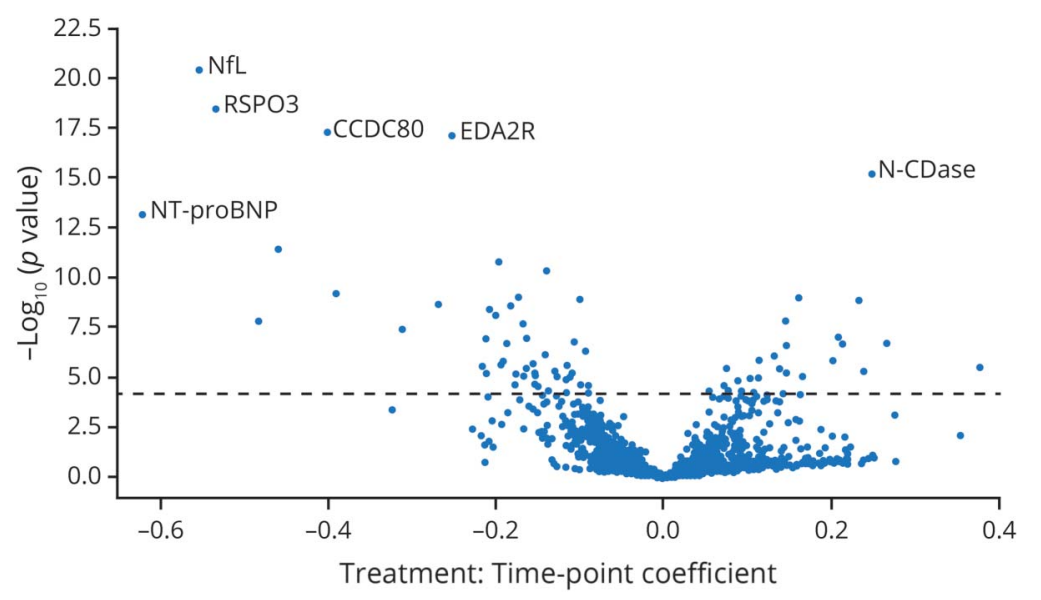

Proteins are shown here as a volcano plot, with the strength of the association on the $y$-axis $\left(-\log _{10}[p\right.$ value]) and the effect size on the $x$-axis (shown as the treatment $\times$ time-point coefficient from the model). A full list of the 66 most enriched proteins can be found in table e-1, doi.org/10.5061/dryad. 1 vhhmgqqk. The gray dotted line represents the Bonferroni multiple testing-corrected $p$ value threshold above which proteins are considered significant. CCDC80 = coiled-coil domain containing 80; EDA2R = ectodysplasin $\mathrm{A} 2$ receptor: $\mathrm{N}$-CDase $=$ neutral ceramidase; $\mathrm{NfL}=$ neurofilament light chain; NT-proBNP = N-terminal prohormone of brain-type natriuretic peptide; RSPO3 = R-spondin 3.

controls (figure 3B). At 18 months, it was found that plasma proteomes of patients treated with patisiran were more similar to those of healthy controls compared with patients treated with placebo (figure $3 \mathrm{C}$ ). Because there is significant heterogeneity in the proteome of patients at baseline, individual patient trajectories were also captured

Figure 3 Gobal Changes in Plasma Proteomes Observed With the Proteome of Patients Treated With Patisiran Trending Toward That of Healthy Controls at 18 Months

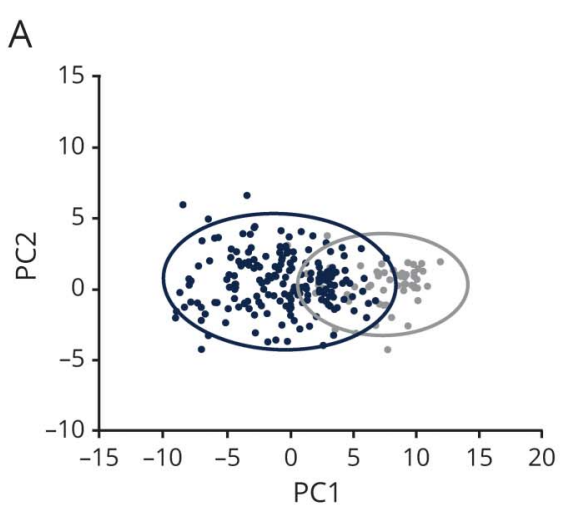

D
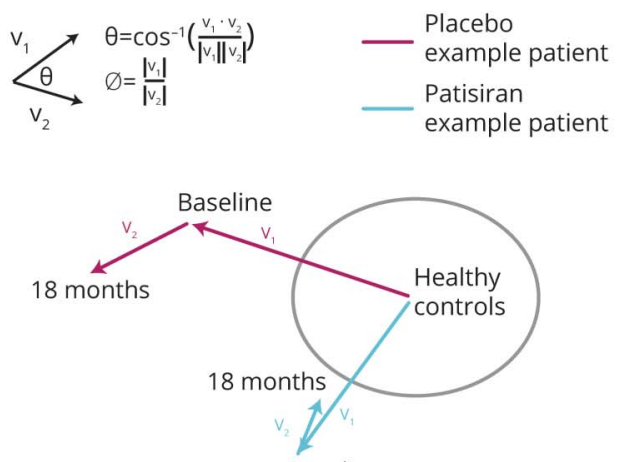

Baseline
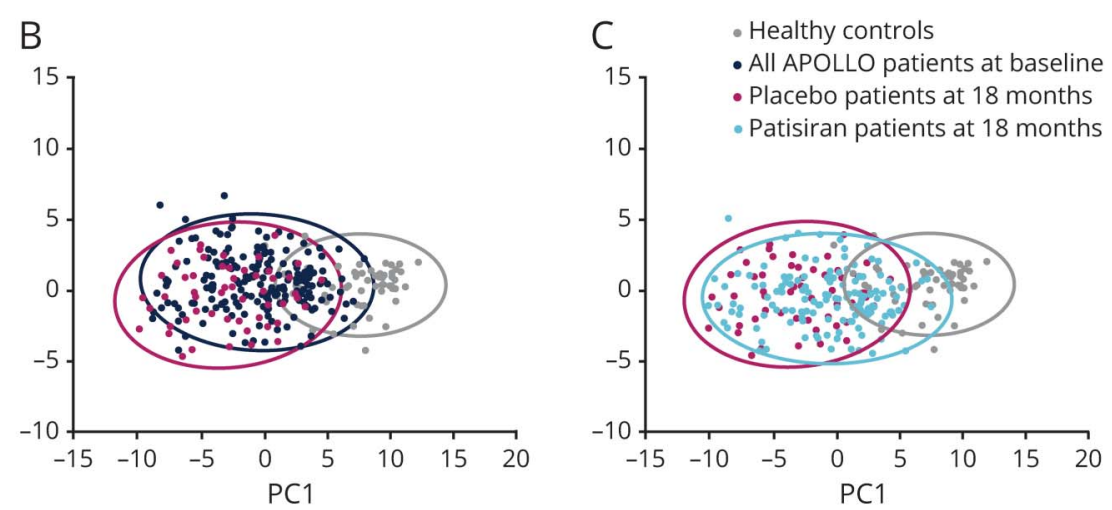

$\mathrm{E}$

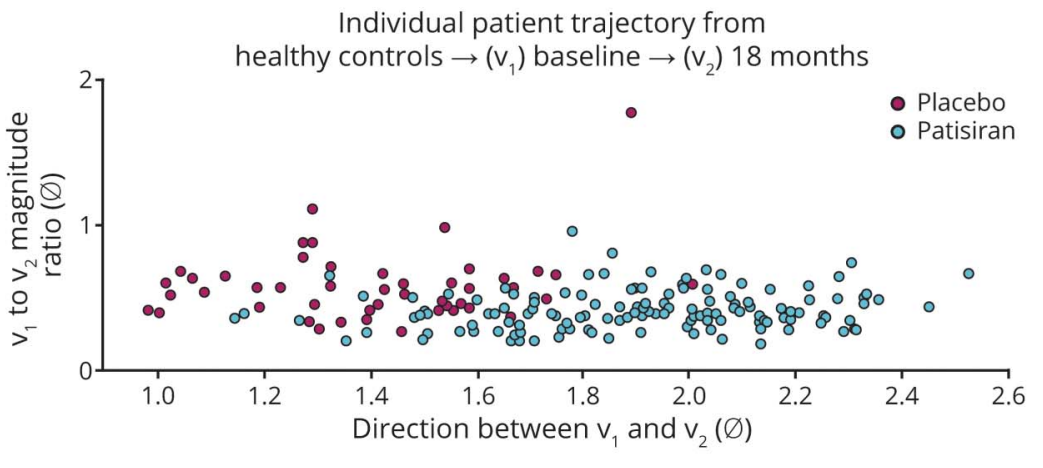

(A) The 66 most changed proteins from the previous analysis were used to project the differences between patients with hereditary transthyretin-mediated amyloidosis and healthy controls at baseline onto 2 principal components (PC1 and PC2) that most explained the difference in the data sets; analysis of (B) patients treated with placebo at 18 months and (C) patients treated with patisiran at 18 months is shown in the same PC1 and PC2 space. (D) Illustrative diagram depicting $v_{1}$ and $v_{2}$ as well as $\phi$ and $\theta$. For each patient, a vector $\left(v_{1}\right)$ from the mean healthy control to baseline and a second vector $\left(v_{2}\right)$ from baseline to month 18 were used to compute 2 metrics, 1 for the ratio of the magnitude of $v_{2}$ compared with $v_{1}$, denoted as $\phi$, and $\theta$ as the angle between the 2 vectors. Here $\varphi$ measures the rate of disease progression or reversal and $\theta$ measures the directionality of the proteome (defined by whether the proteome moves away or toward the healthy controls). (E) Individual patient trajectories are shown, separated by whether patients were on placebo or patisiran treatment. APOLLO = Phase 3 Study of Patisiran for the Treatment of Hereditary Transthyretin-Mediated Amyloidosis With Polyneuropathy. 


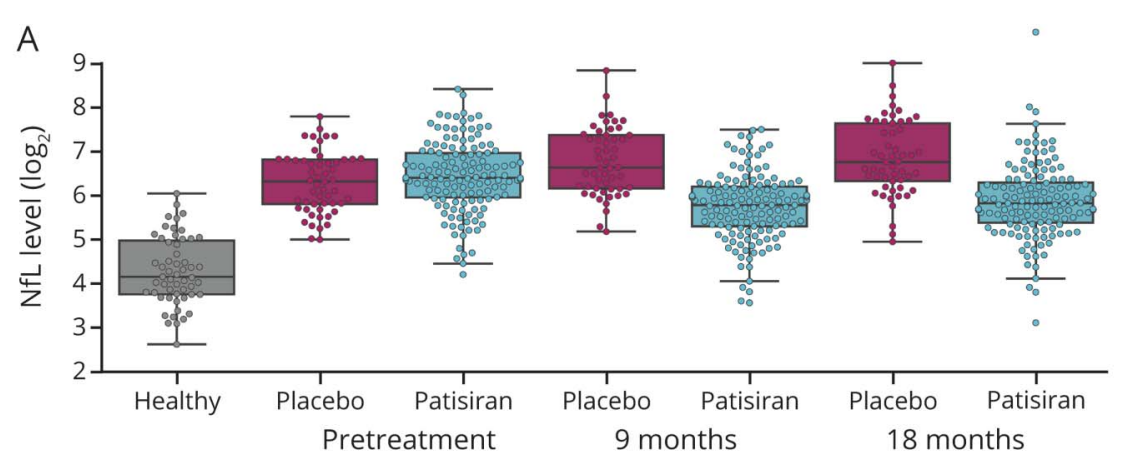

$B$

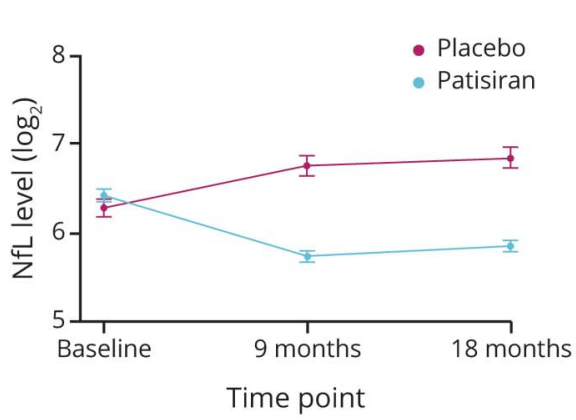

C

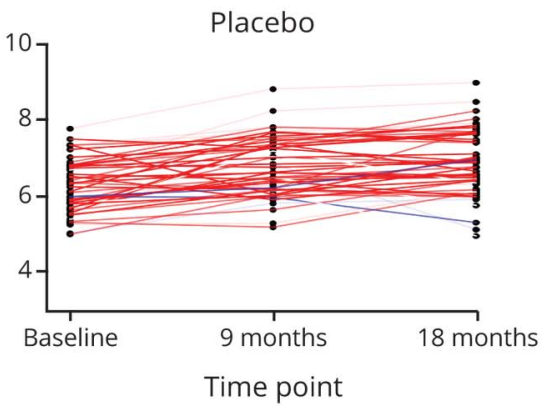

D

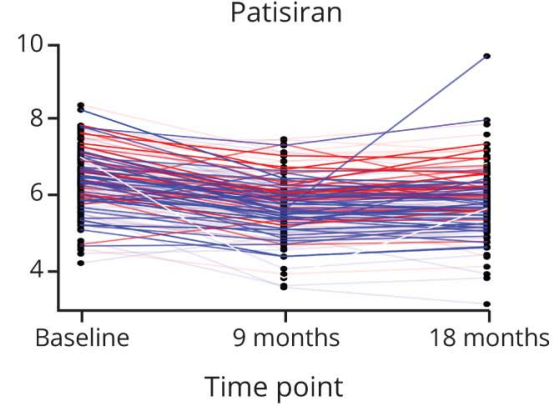

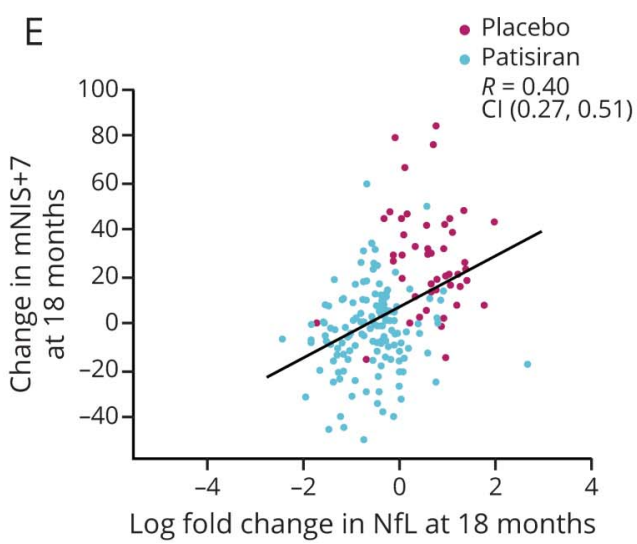

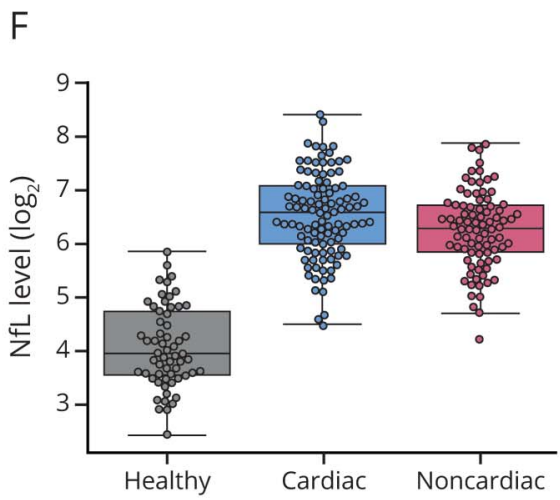

(A) Levels of neurofilament light chain (NfL) (normalized protein expression values, which are on a log $\mathrm{Scale}_{2}$ in healthy controls and patients treated with placebo or patisiran at baseline, 9 months, or 18 months. Boxplots show the first quartile, median, and third quartile of the data; the whiskers are the minimum and maximum values within 1.5× the interquartile range. Group comparisons can be found in table e-2, doi.org/10.5061/dryad.1vhhmgqqk. (B) Mean \pm standard error of NfL levels in patients treated with patisiran or placebo at baseline and 9 and 18 months. Trajectories of individual patients on (C) placebo or (D) patisiran over time, color-coded by their corresponding worsening (change in modified Neuropathy Impairment Score +7 [mNIS+7] score >0; red) or improvement (change in $\mathrm{mNIS}+7$ score <0; blue) in mNIS+7 score from baseline to 18 months. (E) Correlation between change in NfL levels from baseline to 18 months and the corresponding change in mNIS+7 colored by treatment. (F) Levels of NfL separated by whether patients were in the predefined cardiac subpopulation of Study of an Investigational Drug, Patisiran (ALN-TTR02), for the Treatment of Transthyretin (TTR)-Mediated Amyloidosis (APOLLO) or not, at baseline (table e-4, doi.org/10.5061/dryad.1vhhmgqqk). Boxplots show the first quartile, median, and third quartile of the data; the whiskers are the minimum and maximum values within $1.5 \times$ the interquartile range. $\mathrm{Cl}=$ confidence interval.

in 66-dimensional space. For each patient, the course of their disease progression or reversal was defined with 2 metrics, the first representing the rate $(\varphi)$ and a second representing whether the proteome moved away from or toward the healthy controls $(\theta$; see figure $3 \mathrm{D}$ legend). Plotting these 2 parameters clearly separated patients treated with patisiran and patients treated with placebo (figure $3 \mathrm{E}$ ), emphasizing the distinct differences in the proteomes of the 2 groups at 18 months.
Plasma NfL is Increased in Patients With ATTRv Amyloidosis With Polyneuropathy and Decreases With Patisiran Treatment

$\mathrm{NfL}$ was the most significantly changed protein in the analysis when comparing patients treated with placebo and patisiran with ATTRv amyloidosis with polyneuropathy and was therefore investigated in more detail. Patients diagnosed with ATTRv amyloidosis with polyneuropathy had >4-fold higher levels of 

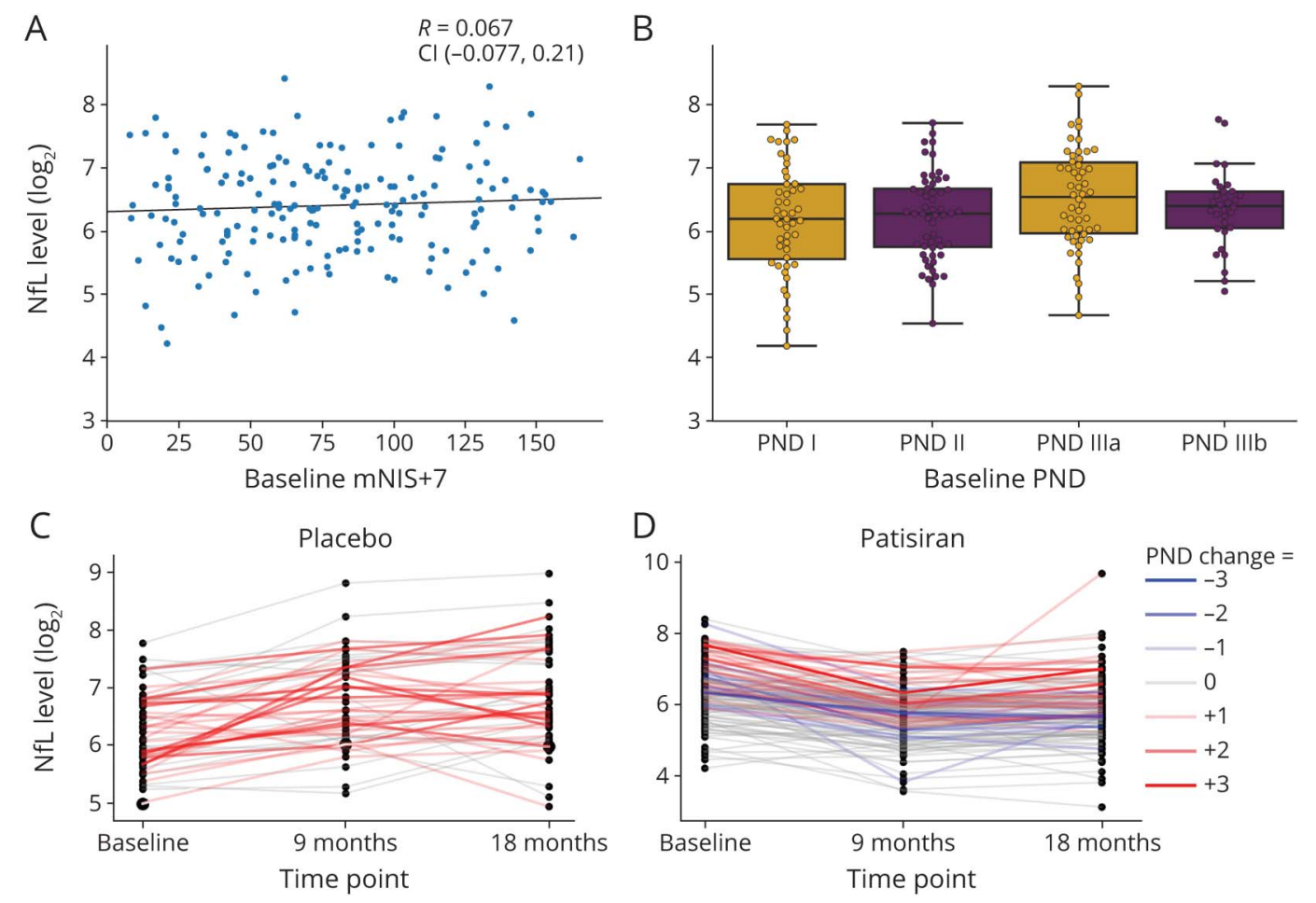

Associations as measured by (A) modified Neuropathy Impairment Score +7 (mNIS+7) and (B) polyneuropathy disability (PND) are not significant (table e-3, doi.org/10.5061/dryad.1vhhmgqqk). Trajectories of individual patients on (C) placebo or (D) patisiran over time, color-coded by their corresponding worsening (change in PND >0; red), no change (gray), and improvement (change in PND <0; blue) in PND score from baseline to 18 months. Boxplots show the first quartile, median, and third quartile of the data; the whiskers are the minimum and maximum values within $1.5 \times$ the interquartile range. $\mathrm{Cl}=$ confidence interval; NfL = neurofilament light chain; $R=$ Pearson correlation coefficient estimate.

$\mathrm{NfL}$ in their plasma at baseline relative to healthy controls, and this result held up independently of age adjustment (figure 4A; $\log _{2}$ scale; table e-2, doi.org/10.5061/dryad.1vhhmgqqk). As expected, plasma NfL levels at baseline did not differ between the patisiran and placebo groups. The patisirantreated group showed a significant decline in plasma $\mathrm{NfL}$ levels at 9 months that was sustained at 18 months, whereas plasma NfL levels in the placebo group increased at 9 months relative to baseline, and this level was sustained at 18 months (figure 4B). At 18 months, patients treated with placebo had 2-fold higher NfL plasma levels than patients treated with patisiran. Treatment with patisiran significantly lowered NfL levels in patients with ATTRv amyloidosis toward levels observed in healthy controls.

To assess variability between individuals, plasma NfL levels were plotted over time for each patient receiving placebo (figure 4C) or patisiran (figure 4D) treatment. Consistent with the general trend in the previous analysis, patients receiving placebo showed increasing plasma NfL levels over time, whereas patients receiving patisiran demonstrated decreasing plasma NfL levels. Changes in plasma NfL levels were correlated with changes in $\mathrm{mNIS}+7$ score at 18 months $(R=0.4$ [95\% CI 0.27-0.51]; figure 4E), indicating that decreasing plasma NfL levels are associated with an improvement in polyneuropathy. Notably, no correlation was observed between plasma NfL levels and $\mathrm{mNIS}+7(R=0.07$, age adjusted $R=0.1)$ or PND score at baseline (table e-3, doi.org/10.5061/dryad. 1vhhmgqqk), regardless of age adjustment, although changes in PND score over time also trended with changes in NfL levels over time (figure 5). In addition, patients belonging to the prespecified cardiac subpopulation of the APOLLO study (defined as baseline left ventricular wall thickness $\geq 13 \mathrm{~mm}$ in the absence of a history of aortic valve disease or hypertension $)^{6}$ saw significant elevation of plasma NfL levels (figure 4F and table e-4, doi.org/10.5061/dryad.1vhhmgqqk) relative to healthy controls, as did patients who did not belong to this prespecified cardiac subpopulation. This suggests that NfL can serve as a biomarker of polyneuropathy in patients regardless of whether they meet specified criteria for cardiac involvement.

The initial NfL measurements were validated with a widely used ultrasensitive single molecule quantitative assay to provide absolute plasma concentrations of NfL in healthy controls and ATTRv amyloidosis patient samples from APOLLO at baseline and 18 months (figure 6 and table e-5, doi.org/10.5061/dryad. 1vhhmgqqk). There was a strong correlation between the 2 assays used to measure plasma NfL levels $(R=0.96[95 \% \mathrm{CI}$ 0.96-0.97]; figure 6A). With the use of the quantitative assay, the mean NfL level in healthy controls was $16.3 \mathrm{pg} / \mathrm{mL}$, consistent with published reports (figure 6B). ${ }^{16}$ Patients with ATTRv amyloidosis with polyneuropathy had plasma NfL 
Figure 6 Quantitative Measurement of NfL Confirm Previous Findings and Show Potential of Plasma NfL Levels to Distinguish Between Healthy Controls and Patients With ATTRv Amyloidosis With Polyneuropathy

A

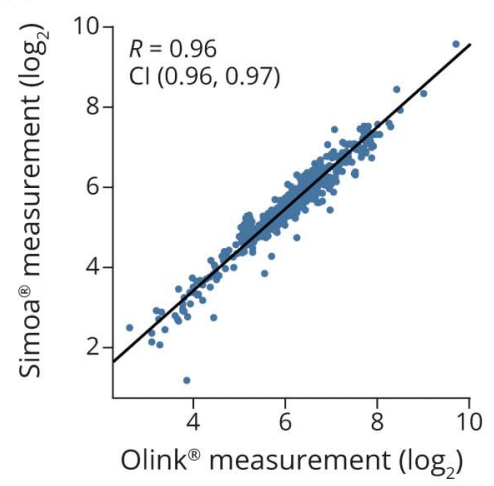

C

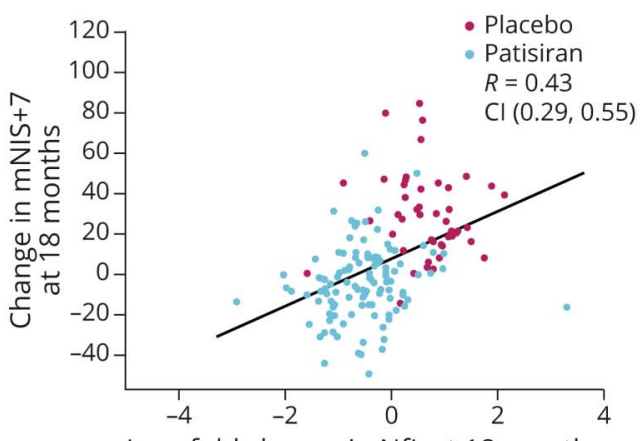

$\log _{2}$ fold change in NfL at 18 months

E

$\mathrm{E}$

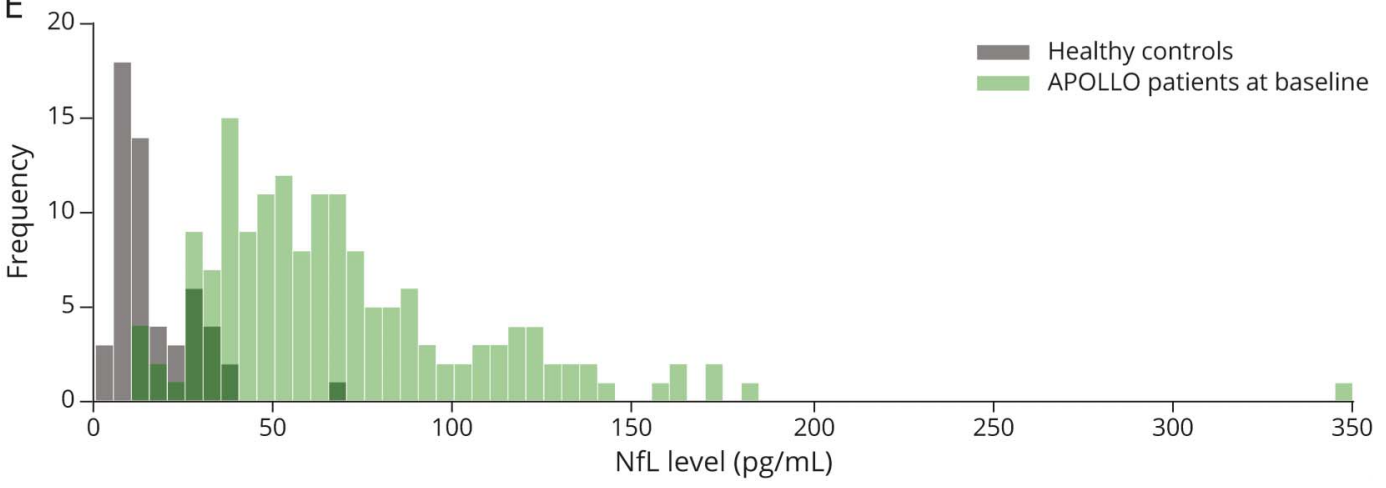

B

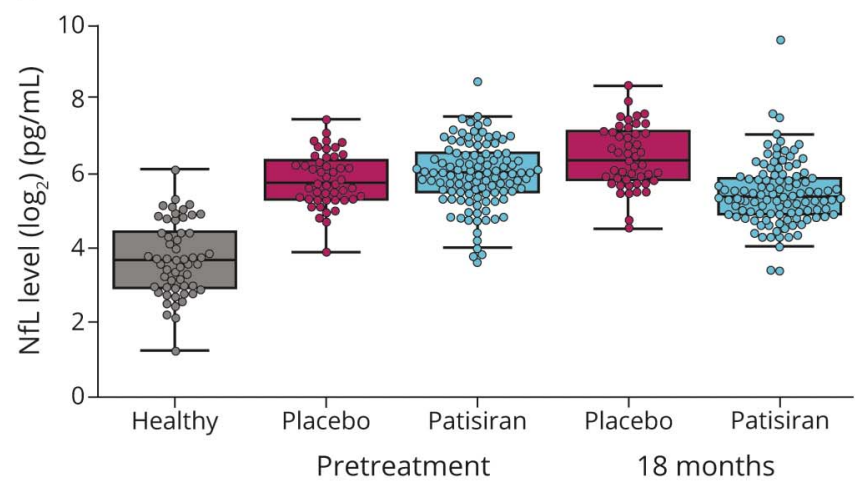

D

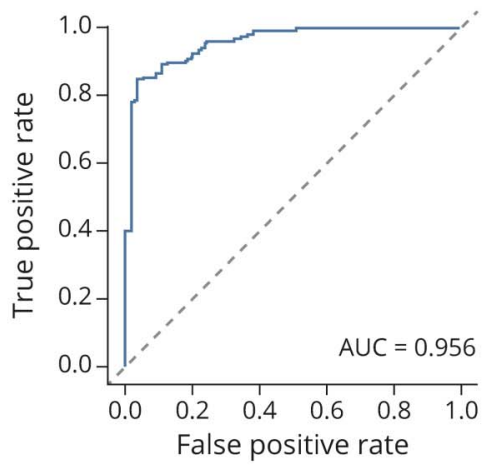

A) Correlation between Olink and Quanterix Simoa platforms is 0.96. Group comparisons can be found in table e-5, doi.org/10.5061/dryad.1vhhmgqqk. (B) Levels of neurofilament light chain (NfL) in healthy controls and patients treated with placebo or patisiran at baseline or 18 months. Boxplots show the first quartile, median, and third quartile of the data; the whiskers are the minimum and maximum values within $1.5 \times$ the interquartile range. (C) Correlation between change in NfL levels from baseline to 18 months and the corresponding change in modified Neuropathy Impairment Score +7 (mNIS+7) score colored by treatment. (D) Receiver operator characteristic curve analysis of NfL plasma levels in healthy controls vs patients with hereditary transthyretinmediated amyloidosis with polyneuropathy. The area under the curve (AUC) is 0.956. (E) Histograms showing the distributions of NfL concentrations in healthy controls (dark gray) and patients with ATTRv amyloidosis at baseline (green). APOLLO = Phase 3 Study of Patisiran for the Treatment of Hereditary Transthyretin-Mediated Amyloidosis With Polyneuropathy.

levels that were $\approx 4$-fold higher at baseline relative to healthy controls (69.4 pg/mL, effect size 53.1 [95\% CI 45.9-60.5]), which, after 18 months, decreased after patisiran treatment (48.8 pg/mL, effect size -23.2 [95\% CI -33.4 to -13.1$]$ ) and increased in the placebo group $(99.5 \mathrm{pg} / \mathrm{mL}$, effect size 36.3 [95\% CI 16.5-56.1]). One outlier with NfL plasma levels of $747 \mathrm{pg} / \mathrm{mL}$ in the patisiran-treated group was excluded from the calculations (figure 6, B and E) because this individual experienced a study-unrelated cerebral infarct at month 17 that may have caused the observed elevations of NfL. Consistent with the initial plasma NfL measurements (figure 4), measurements using the quantitative assay demonstrated a strong correlation between change in mNIS+7 score and change in plasma NfL levels at 18 months for each patient $(R=0.43[95 \%$ CI 0.29-0.55]; figure 6C). Receiver operating characteristic analysis determined that plasma NfL levels could discriminate between healthy controls and patients with ATTRv amyloidosis with polyneuropathy (area under the curve 0.956; figure 
$6 \mathrm{D}$ ), and the distribution of each was plotted (figure 6E). With the current data, an NfL level of $37 \mathrm{pg} / \mathrm{mL}$ may distinguish between healthy controls and patients with diagnosed ATTRv amyloidosis with polyneuropathy, with a false-positive rate of $3.6 \%$ and true-positive rate of $84.9 \%$.

\section{Discussion}

Plasma proteomics and the identification of minimally invasive biomarkers are emerging as integral parts of modern drug discovery and clinical development. In an effort to leverage this approach, the plasma proteomes of patients with ATTRv amyloidosis with polyneuropathy were investigated over time in the largest clinical proteomic study of this disease to date. Proteomic analyses of patient plasma samples demonstrated that patisiran treatment resulted in a general shift of patients' proteomes toward those of healthy controls compared with patients who received placebo treatment. This finding suggests that the plasma protein milieu is reflective of ATTRv amyloidosis and response to treatment. Across $>1,000$ unique proteins that were assessed from plasma samples collected in the APOLLO study, 66 proteins were found to exhibit a significantly different plasma level time profile in patients treated with patisiran compared with patients treated with placebo over the course of 18 months. Of these, NfL was identified as the protein most significantly different between the 2 groups. Outside of NfL, NT-proBNP, and hepatocyte growth factor, none of the remaining proteins have been characterized as a biomarker of neuronal injury or cardiac disease. Hepatocyte growth factor elevations have been described in AL amyloidosis and, to a lesser extent, in ATTRv amyloidosis with cardiomyopathy. ${ }^{17}$ Further studies of these proteins may help further elucidate the multisystem manifestations of ATTRv amyloidosis. These results are consistent with raised plasma NfL levels recently observed in patients with ATTRv amyloidosis compared with healthy controls. ${ }^{18,19}$ Collectively the findings demonstrate that patient proteome changes are detectable after treatment and support the identification of proteins that both have the potential to serve as biomarkers and offer insights into ATTRv amyloidosis disease biology.

Of highest clinical interest are the high plasma NfL levels detected in patients with ATTRv amyloidosis with polyneuropathy and the subsequent decrease of these after patisiran treatment. This is important given that NfL is an integral component of the axonal structure of neurons, and the neuropathy observed with ATTRv amyloidosis is often characteristic of axonal injury ${ }^{20}$ with deposition of amyloid fibrils that cause microangiopathy and atrophy of Schwann cells. ${ }^{1} \mathrm{NfL}$ has been described extensively as a biomarker of neuroaxonal injury across many CNS diseases, including multiple sclerosis, ${ }^{21}$ Alzheimer disease, ${ }^{22}$ and Huntington disease, ${ }^{23}$ as well as peripheral nervous system diseases such as vasculitis, ${ }^{24}$ chronic inflammatory demyelinating neuropathy, ${ }^{25}$ Guillain-Barré syndrome, ${ }^{26}$ and Charcot-Marie-Tooth disease (CMT). ${ }^{27}$
Mutations in NfL itself can also lead to a specific type of CMT disease (CMT2E), indicating that NfL plays a crucial role in peripheral nerve function. ${ }^{28}$ Elevated NfL levels in these diseases are thought to be caused by the release of $\mathrm{NfL}$ into the circulation from damaged neurons, ${ }^{29}$ and it has been identified in both $\mathrm{CSF}^{30}$ and blood. ${ }^{31}$ In this study, the mean NfL levels described in patients with ATTRv amyloidosis with polyneuropathy $(69.4 \mathrm{pg} / \mathrm{mL})$ were higher than those observed for other peripheral nerve disorders (chronic inflammatory demyelinating neuropathy $42 \mathrm{pg} / \mathrm{mL}$, CMT $26 \mathrm{pg} / \mathrm{mL})^{25,27}$ and consistent with a previous report in patients with ATTRv amyloidosis $(58.1 \mathrm{pg} / \mathrm{mL}){ }^{19}$

Patisiran treatment lowers TTR messenger RNA levels (and therefore circulating TTR protein), potentially resulting in a reduction of amyloid burden, which is hypothesized to halt or reduce neuronal damage. ${ }^{14}$ This hypothesis was supported by the results of the APOLLO study in which patisiran halted the progression of polyneuropathy and reversed polyneuropathy from baseline in the majority of patients (least-squares mean difference -6.0), whereas mNIS+7 score worsened from baseline in patients treated with placebo (least-squares mean difference +28.0). ${ }^{6}$ The current study suggests that a patisiranmediated reduction in nerve damage also causes a reduction in plasma levels of NfL. Reductions in NfL levels were observed during the first 9 months of patisiran treatment and were sustained for a further 9 months. This response may suggest that patisiran treatment reduces ATTRv amyloidosisassociated neuronal damage within a short time frame and subsequently maintains this effect, although further studies are needed to confirm and further characterize this finding.

The correlation observed between change in mNIS+7 score and change in plasma NfL levels over 18 months indicates that decreasing levels of NfL detected in patients treated with patisiran are also associated with improvement in polyneuropathy. There was, however, no correlation between NfL levels and mNIS +7 scores at baseline. One explanation for this observation is that significant heterogeneity exists in interpatient plasma NfL levels, while intrapatient NfL changes over time may be more informative regarding changes in disease status. Another possibility is that NfL reflects active or continuing neuronal damage at a specific point in time, whereas mNIS+7 measures the overall burden of neurologic impairment. Because NfL is a biologic indicator of nerve damage while mNIS+7 captures the clinical impact of this damage, a perfect correlation would not be expected.

Notably, levels of NfL were elevated at baseline in patients with and without evidence of cardiac amyloid involvement, suggesting that NfL may be useful as a biomarker of polyneuropathy in patients previously diagnosed with ATTRv amyloidosis with cardiomyopathy. An investigation of plasma $\mathrm{NfL}$ levels in patients with predominantly cardiomyopathic phenotype may further support ATTRv amyloidosis as a single multisystem disease, regardless of TTR mutation or initial presenting symptoms. 
In addition to monitoring polyneuropathy progression and treatment response, NfL may serve as a potential diagnostic biomarker or as an indicator for the transition from an asymptomatic carrier to a symptomatic patient. For example, a recent study of $\mathrm{NfL}$ in asymptomatic carriers of the pathogenic $\mathrm{V} 30 \mathrm{M}$ variant suggests that $\mathrm{NfL}$ levels can distinguish between asymptomatic and symptomatic (as defined by PND $>0$ ) individuals. ${ }^{18}$ The data described here suggest that plasma NfL levels may help provide a clinically meaningful threshold to aid the diagnosis of neuronal damage in ATTRv amyloidosis with polyneuropathy, supplementing clinical assessments that are already being used. While these data suggest that $37 \mathrm{pg} / \mathrm{mL}$ may serve as a potential $\mathrm{NfL}$ level to discriminate presence of disease (with a false positive rate of $3.6 \%$ and true positive rate of $84.9 \%$ ), additional studies are required to define a meaningful diagnostic threshold that can be used in clinical practice. Due to the heterogeneity observed in patients with ATTRv amyloidosis with polyneuropathy, the amount of change in NfL levels over time may prove to be a more sensitive indicator of neuronal damage than absolute levels. In plasma samples collected from the Phase 3 APOLLO study, an average increase of $30 \mathrm{pg} / \mathrm{mL}$ was observed in the placebo group over the course of 18 months, indicating that $\mathrm{NfL}$ may also serve as a biomarker of polyneuropathy progression.

Further studies in larger patient cohorts, including asymptomatic carriers of ATTRv amyloidosis, and longer follow-up are needed to confirm and expand on these early findings. Additional longitudinal time points would aid in further understanding the dynamics of NfL and how they relate temporally with mNIS+7 score in individual patients over time. Because NfL levels are known to increase with age, it is possible that an improved threshold for evidence of disease may be age dependent. The small size of the healthy control group in this study did not permit accurate estimation of healthy control NfL levels at all ages; therefore, an age-corrected threshold was not proposed here.

This study represents the first system-wide proteomics interrogation of response to an RNAi therapeutic in humans. The analysis of samples collected from APOLLO patients has led to an improved understanding of polyneuropathy disease progression in patients with ATTRv amyloidosis. NfL has been identified as a protein that is differentially expressed in patients with ATTRv amyloidosis with polyneuropathy vs healthy controls. Changes in plasma NfL levels are also seen in response to patisiran treatment over time, highlighting the potential of this protein to serve as a biomarker in several capacities, including as an early diagnostic biomarker of ATTRv amyloidosis or a predictor of when disease may become manifest in asymptomatic carriers of pathogenic TTR mutations, and as an objective marker for monitoring polyneuropathy disease progression or reversal over time. The results from this comprehensive proteomic analysis using samples from a placebo-controlled study have (1) identified $\mathrm{NfL}$ as a potential multipurpose polyneuropathy biomarker for ATTRv amyloidosis, (2) provided new insights into
ATTRv amyloidosis disease biology, and (3) enabled the first system-wide proteomics interrogation of response to an RNAi therapeutic in humans.

\section{Acknowledgment}

The authors thank the patients and their families involved in the APOLLO study for their valued contribution to this study. This study was funded by Alnylam Pharmaceuticals Inc., USA. Editorial support was provided by Ed Childs, $\mathrm{PhD}$, of Adelphi Communications Ltd, Macclesfield, UK, in accordance with the Good Publication Practice guidelines, funded by Alnylam Pharmaceuticals Inc, USA.

\section{Study Funding}

The study was sponsored by Alnylam Pharmaceuticals.

\section{Disclosure}

S. Ticau, G.V. Sridharan, S. Tsour, W.L. Cantley, A. Chan, J.A. Gilbert, D. Erbe, E. Aldinc, K. Fitzgerald, A. Vaishnaw, and P. Nioi are employed by Alnylam Pharmaceuticals and report ownership of shares in Alnylam Pharmaceuticals. M.M. Reilly has participated in a clinical trial sponsored by Ionis and has received consulting fees from Alnylam Pharmaceuticals, Ionis, and Akcea. D. Adams has participated in clinical trials sponsored by Alnylam Pharmaceuticals and Ionis and received consulting fees advisory from Alnylam Pharmaceuticals Pharmaceuticals and Pfizer. M. Polydefkis has participated in clinical trials sponsored by Alnylam, Ionis, and Pfizer and has received consulting fees from Alnylam Pharmaceuticals, Ionis, and Pfizer. Go to Neurology.org/N for full disclosures.

\section{Publication History}

Received by Neurology April 9, 2020. Accepted in final form September 1, 2020.

Appendix Authors

\begin{tabular}{|c|c|c|}
\hline Name & Location & Contribution \\
\hline $\begin{array}{l}\text { Simina } \\
\text { Ticau, PhD }\end{array}$ & $\begin{array}{l}\text { Alnylam Pharmaceuticals, } \\
\text { Cambridge, MA }\end{array}$ & $\begin{array}{l}\text { Designed the study; } \\
\text { analyzed and interpreted the } \\
\text { data; performed statistical } \\
\text { analysis; created the figures; } \\
\text { and wrote the report }\end{array}$ \\
\hline $\begin{array}{l}\text { Gautham } \\
\text { V. } \\
\text { Sridharan, } \\
\text { PhD }\end{array}$ & $\begin{array}{l}\text { Alnylam Pharmaceuticals, } \\
\text { Cambridge, MA }\end{array}$ & $\begin{array}{l}\text { Designed the study; } \\
\text { analyzed and interpreted the } \\
\text { data; performed statistical } \\
\text { analysis; created the figures; } \\
\text { and wrote the report }\end{array}$ \\
\hline $\begin{array}{l}\text { Shira } \\
\text { Tsour, MS }\end{array}$ & $\begin{array}{l}\text { Alnylam Pharmaceuticals, } \\
\text { Cambridge, MA }\end{array}$ & $\begin{array}{l}\text { Designed the study; } \\
\text { analyzed and interpreted } \\
\text { the data; created the } \\
\text { figures; and wrote the } \\
\text { report }\end{array}$ \\
\hline $\begin{array}{l}\text { William L. } \\
\text { Cantley, } \\
\text { PhD }\end{array}$ & $\begin{array}{l}\text { Alnylam Pharmaceuticals, } \\
\text { Cambridge, MA }\end{array}$ & $\begin{array}{l}\text { Interpreted the data and } \\
\text { wrote the report }\end{array}$ \\
\hline $\begin{array}{l}\text { Amy Chan, } \\
\text { PhD }\end{array}$ & $\begin{array}{l}\text { Alnylam Pharmaceuticals, } \\
\text { Cambridge, MA }\end{array}$ & $\begin{array}{l}\text { Designed the study; } \\
\text { interpreted the data; and } \\
\text { wrote the report }\end{array}$ \\
\hline
\end{tabular}

Continued 
Appendix (continued)

\begin{tabular}{|c|c|c|}
\hline Name & Location & Contribution \\
\hline $\begin{array}{l}\text { Jason A. } \\
\text { Gilbert, MS }\end{array}$ & $\begin{array}{l}\text { Alnylam Pharmaceuticals, } \\
\text { Cambridge, MA }\end{array}$ & $\begin{array}{l}\text { Interpreted the data and } \\
\text { wrote the report }\end{array}$ \\
\hline $\begin{array}{l}\text { David Erbe, } \\
\text { PhD }\end{array}$ & $\begin{array}{l}\text { Alnylam Pharmaceuticals, } \\
\text { Cambridge, MA }\end{array}$ & $\begin{array}{l}\text { Interpreted the data and } \\
\text { wrote the report }\end{array}$ \\
\hline $\begin{array}{l}\text { Emre } \\
\text { Aldinc, MD }\end{array}$ & $\begin{array}{l}\text { Alnylam Pharmaceuticals, } \\
\text { Cambridge, MA }\end{array}$ & $\begin{array}{l}\text { Interpreted the data and } \\
\text { wrote the report }\end{array}$ \\
\hline $\begin{array}{l}\text { Mary M. } \\
\text { Reilly, MD }\end{array}$ & $\begin{array}{l}\text { MRC Centre for } \\
\text { Neuromuscular Diseases, } \\
\text { UCL Queen Square } \\
\text { Institute of Neurology, } \\
\text { London, UK }\end{array}$ & $\begin{array}{l}\text { Interpreted the data and } \\
\text { wrote the report }\end{array}$ \\
\hline $\begin{array}{l}\text { David } \\
\text { Adams, MD }\end{array}$ & $\begin{array}{l}\text { AP-HP. Université Paris- } \\
\text { Saclay, CHU Bicêtre, } \\
\text { INSERM U1195, Le Kremlin } \\
\text { Bicêtre, France }\end{array}$ & $\begin{array}{l}\text { Interpreted the data and } \\
\text { wrote the report }\end{array}$ \\
\hline $\begin{array}{l}\text { Michael } \\
\text { Polydefkis, } \\
\text { MD }\end{array}$ & $\begin{array}{l}\text { Department of Neurology, } \\
\text { Johns Hopkins University } \\
\text { School of Medicine, } \\
\text { Baltimore, MD }\end{array}$ & $\begin{array}{l}\text { Interpreted the data and } \\
\text { wrote the report }\end{array}$ \\
\hline $\begin{array}{l}\text { Kevin } \\
\text { Fitzgerald, } \\
\text { PhD }\end{array}$ & $\begin{array}{l}\text { Alnylam Pharmaceuticals, } \\
\text { Cambridge, MA }\end{array}$ & $\begin{array}{l}\text { Designed the study; } \\
\text { interpreted the data; and } \\
\text { wrote the report }\end{array}$ \\
\hline $\begin{array}{l}\text { Akshay } \\
\text { Vaishnaw, } \\
\text { MD }\end{array}$ & $\begin{array}{l}\text { Alnylam Pharmaceuticals, } \\
\text { Cambridge, MA }\end{array}$ & $\begin{array}{l}\text { Designed the study; } \\
\text { interpreted the data; and } \\
\text { wrote the report }\end{array}$ \\
\hline $\begin{array}{l}\text { Paul Nioi, } \\
\text { PhD }\end{array}$ & $\begin{array}{l}\text { Alnylam Pharmaceuticals, } \\
\text { Cambridge, MA }\end{array}$ & $\begin{array}{l}\text { Designed the study; } \\
\text { interpreted the data; and } \\
\text { wrote the report }\end{array}$ \\
\hline
\end{tabular}

\section{References}

1. Adams D, Koike H, Slama M, Coelho T. Hereditary transthyretin amyloidosis: a model of medical progress for a fatal disease. Nat Rev Neurol 2019;15:387-404

2. Hawkins PN, Ando Y, Dispenzeri A, Gonzalez-Duarte A, Adams D, Suhr OB Evolving landscape in the management of transthyretin amyloidosis. Ann Med 2015; 47:625-638.

3. Conceição I, Gonzalez-Duarte A, Obici L, et al. "Red-flag" symptom clusters in transthyretin familial amyloid polyneuropathy. J Peripher Nerv Syst 2016;21:5-9.

4. Rapezzi C, Quarta CC, Obici L, et al. Disease profile and differential diagnosis of hereditary transthyretin-related amyloidosis with exclusively cardiac phenotype: an Italian perspective. Eur Heart J 2013;34:520-528.

5. Coelho T, Maurer MS, Suhr OB. THAOS-the Transthyretin Amyloidosis Outcomes Survey: initial report on clinical manifestations in patients with hereditary and wild-type transthyretin amyloidosis. Curr Med Res Opin 2013;29:63-76.

6. Adams D, Gonzalez-Duarte A, O’Riordan WD, et al. Patisiran, an RNAi therapeutic, for hereditary transthyretin amyloidosis. N Engl J Med 2018;379:11-21.
7. Adams D, Suhr OB, Hund E, et al. First European consensus for diagnosis, management, and treatment of transthyretin familial amyloid polyneuropathy. Curr Opin Neurol 2016;29(suppl 1):S14-S26.

8. Obici L, Kuks JB, Buades J, et al. Recommendations for presymptomatic genetic testing and management of individuals at risk for hereditary transthyretin amyloidosis. Curr Opin Neurol 2016;29(suppl 1):S27-S35.

9. Parman Y, Adams D, Obici L, et al. Sixty years of transthyretin familial amyloid polyneuropathy (TTR-FAP) in Europe: where are we now? A European network approach to defining the epidemiology and management patterns for TTR-FAP. Cur Opin Neurol 2016;29(suppl 1):S3-S13.

10. Swiecicki PL, Zhen DB, Mauermann ML, et al. Hereditary ATTR amyloidosis: a single-institution experience with 266 patients. Amyloid 2015;22:123-131.

11. Adams D, Suhr OB, Dyck PJ, et al. Trial design and rationale for APOLLO, a phase 3, placebo-controlled study of patisiran in patients with hereditary ATTR amyloidosis with polyneuropathy. BMC Neurol 2017;17:181.

12. Chan GG, Koch CM, Connors LH. Blood proteomic profiling in inherited (ATTRm) and acquired (ATTRwt) forms of transthyretin-associated cardiac amyloidosis. J Proteome Res 2017; 16:1659-1668.

13. Chan GG, Koch CM, Connors LH. Serum proteomic variability associated with clinical phenotype in familial transthyretin amyloidosis (ATTRm). J Proteome Res 2017;16:4104-4112.

14. Coelho T, Adams D, Silva A, et al. Safety and efficacy of RNAi therapy for transthyretin amyloidosis. N Engl J Med 2013;369:819-829.

15. Dyck PJB, González-Duarte A, Obici L, et al. Development of measures of polyneuropathy impairment in hATTR amyloidosis: from NIS to mNIS+7. J Neurol Sci 2019;405:116424.

16. Disanto G, Barro C, Benkert P, et al. Serum neurofilament light: a biomarker of neuronal damage in multiple sclerosis. Ann Neurol 2017;81:857-870.

17. Swiger KJ, Friedman EA, Brittain EL, et al. Plasma hepatocyte growth factor is a novel marker of AL cardiac amyloidosis. Amyloid 2016;23:242-248.

18. Maia LF, Maceski A, Conceição I, et al. Plasma neurofilament light chain: an early biomarker for hereditary ATTR amyloid polyneuropathy. Amyloid 2020;27:97-102.

19. Kapoor M, Foiani M, Heslegrave A, et al. Plasma neurofilament light chain concentration is increased and correlates with the severity of neuropathy in hereditary transthyretin amyloidosis. J Peripher Nerv Syst 2019;24:314-319.

20. Shin SC, Robinson-Papp J. Amyloid neuropathies. Mt Sinai J Med 2012;79:733-748.

21. Gunnarsson $M$, Malmeström C, Axelsson $M$, et al. Axonal damage in relapsing multiple sclerosis is markedly reduced by natalizumab. Ann Neurol 2011;69:83-89.

22. Lewczuk P, Ermann N, Andreasson U, et al. Plasma neurofilament light as a potential biomarker of neurodegeneration in Alzheimer's disease. Alzheimers Res Ther 2018;10:71.

23. Byrne LM, Rodrigues FB, Blennow K, et al. Neurofilament light protein in blood as a potential biomarker of neurodegeneration in Huntington's disease: a retrospective cohort analysis. Lancet Neurol 2017;16:601-609.

24. Bischof A, Manigold T, Barro C, et al. Serum neurofilament light chain: a biomarker of neuronal injury in vasculitic neuropathy. Ann Rheum Dis 2018;77:1093-1094.

25. van Lieverloo GGA, Wieske L, Verhamme C, et al. Serum neurofilament light chain in chronic inflammatory demyelinating polyneuropathy. J Peripher Nerv Syst 2019;24:187-194.

26. Mariotto S, Farinazzo A, Magliozzi R, Alberti D, Monaco S, Ferrari S. Serum and cerebrospinal neurofilament light chain levels in patients with acquired peripheral neuropathies. J Peripher Nerv Syst 2018;23:174-177.

27. Sandelius $\AA$, Zetterberg H, Blennow K, et al. Plasma neurofilament light chain concentration in the inherited peripheral neuropathies. Neurology 2018;90:e518-e524.

28. Jordanova A, De Jonghe P, Boerkoel CF, et al. Mutations in the neurofilament light chain gene (NEFL) cause early onset severe Charcot-Marie-Tooth disease. Brain 2003;126:590-597.

29. Bacioglu M, Maia LF, Preische O, et al. Neurofilament light chain in blood and CSF as marker of disease progression in mouse models and in neurodegenerative diseases. Neuron 2016;91:56-66.

30. Lycke JN, Karlsson JE, Andersen O, Rosengren LE. Neurofilament protein in cerebrospinal fluid: a potential marker of activity in multiple sclerosis. J Neurol Neurosurg Psychiatry 1998;64:402-404.

31. Preische O, Schultz SA, Apel A, et al. Serum neurofilament dynamics predicts neurodegeneration and clinical progression in presymptomatic Alzheimer's disease. Nat Med 2019;25:277-283. 


\section{Neurology}

\section{Neurofilament Light Chain as a Biomarker of Hereditary Transthyretin-Mediated Amyloidosis}

Simina Ticau, Gautham V. Sridharan, Shira Tsour, et al.

Neurology 2021;96; e412-e422 Published Online before print October 21, 2020

DOI 10.1212/WNL.0000000000011090

This information is current as of October 21, 2020

\section{Updated Information \&} Services

References

Citations

Subspecialty Collections

Permissions \& Licensing

Reprints including high resolution figures, can be found at: http://n.neurology.org/content/96/3/e412.full

This article cites 31 articles, 3 of which you can access for free at: http://n.neurology.org/content/96/3/e412.full\#ref-list-1

This article has been cited by 1 HighWire-hosted articles: http://n.neurology.org/content/96/3/e412.full\#\#otherarticles

This article, along with others on similar topics, appears in the following collection(s):

All Medical/Systemic disease

http://n.neurology.org/cgi/collection/all_medical_systemic_disease Class IV

http://n.neurology.org/cgi/collection/class_iv

Peripheral neuropathy

http://n.neurology.org/cgi/collection/peripheral_neuropathy

Information about reproducing this article in parts (figures,tables) or in its entirety can be found online at:

http://www.neurology.org/about/about_the_journal\#permissions

Information about ordering reprints can be found online:

http://n.neurology.org/subscribers/advertise

Neurology ${ }^{\circledR}$ is the official journal of the American Academy of Neurology. Published continuously since 1951, it is now a weekly with 48 issues per year. Copyright Copyright ( 2020 The Author(s). Published by Wolters Kluwer Health, Inc. on behalf of the American Academy of Neurology.. All rights reserved. Print ISSN: 0028-3878. Online ISSN: 1526-632X.

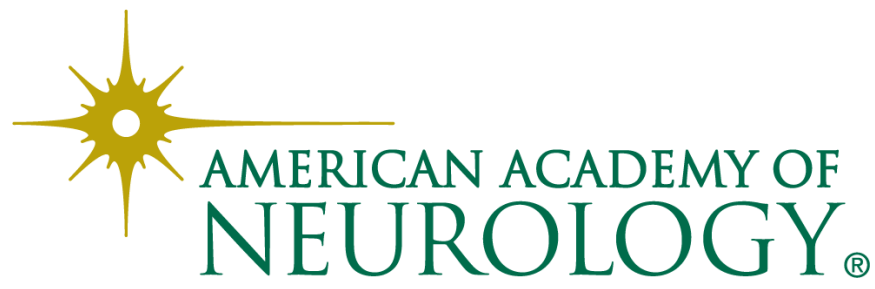

